Published in final edited form as:

Ann Rheum Dis. 2018 June ; 77(6): 861-868. doi:10.1136/annrheumdis-2017-212252.

\title{
Are MRI-detected erosions specific for RA? A large explorative cross-sectional study
}

\author{
Debbie M. Boeters ${ }^{1}$, Wouter P. Nieuwenhuis ${ }^{1}$, Hanna W. van Steenbergen ${ }^{1}$, Monique \\ Reijnierse $^{2}$, Robert B.M. Landewé ${ }^{3}$, and Annette H.M. van der Helm-van Mil1,4 \\ ${ }^{1}$ Department of Rheumatology, Leiden University Medical Center, Leiden, the Netherlands \\ 2Department of Radiology, Leiden University Medical Center, Leiden, the Netherlands \\ ${ }^{3}$ Department of Clinical Rheumatology and Immunology, Amsterdam Medical Center Amsterdam \\ and Atrium Medical Center Heerlen, the Netherlands ${ }^{4}$ Department of Rheumatology, Erasmus \\ University Medical Center, Rotterdam, the Netherlands
}

\section{Abstract}

Objectives-Magnetic resonance imaging (MRI) is recommended in the diagnostic process of rheumatoid arthritis (RA), to detect joint damage early. MRI-detected erosions are also present in symptom-free controls, especially at older age. It is unclear if RA-specific MRI-detected erosions can be distinguished from 'physiological' erosions in symptom-free individuals. This study compared MRI-detected erosions of RA-patients with healthy controls and with other arthritides.

Methods-589 newly-presenting early arthritis patients (238 RA, 351 other arthritides) and 193 symptom-free controls underwent contrast-enhanced 1.5T MRI of unilateral metacarpophalangealand metatarsophalangeal(MTP)-joints. Total erosion score (according to RAMRIS), number, severity, location of erosions and simultaneous presence of MRI-detected inflammation (synovitis and/or bone marrow edema) were compared; participants were categorized in three age-groups $(<40,40-59, \geq 60)$.

Results-RA-patients had statistically significant higher total erosion scores than controls but scores of individual persons largely overlapped. Grade $\geq 2$ erosions and MTP5-erosions were specific for RA (specificity 98-100\% and 90-98\% for different age-groups). MTP1-erosions were only specific if aged $<40$ (specificity $98 \%$ ) and erosions with inflammation if aged $<60$ (specificity

\footnotetext{
Address of correspondence: Debbie M. Boeters, department of Rheumatology C1-R, Leiden University Medical Center PO Box 9600, Leiden 2300RC, the Netherlands, Tel.: +31 71 5265653, Fax: +31 71 5266752, D.M.Boeters@lumc.nl.

Competing Interests

None declared.

Contributorship

DMB, WPN and AHMvdHvM contributed to the conception and study design. DMB analysed the data. DMB, WPN, HWvS, MR, RBML and AHMvdHvM contributed to interpretation of the data. DMB and AHMvdHvM wrote the first version of the manuscript and WPN, HWvS, MR and RBML revised it critically. DMB, WPN, HWvS, MR, RBML and AHMvdHvM read and approved the final manuscript.

Ethical Approval Information

The study on early arthritis patients and the study on healthy controls were both approved by the local medical ethics committee, which is named 'Commissie Medische Ethiek'. All patients signed informed consent.

Data Sharing Statement

Data can be requested from the corresponding author.
} 
91-100\%). $\geq 1$ of the mentioned erosions characteristics were present in $29 \%$ of RA-patients. Comparing RA-patients with other arthritides revealed that grade $\geq 2$ erosions and MTP5-erosions

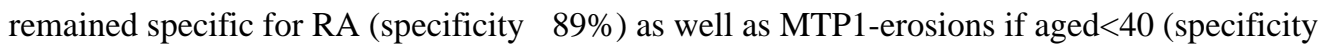
93\%), in contrast to erosions combined with inflammation (specificity 49-85\%).

Conclusions-Total erosion scores of individual persons were largely overlapping. Erosion characteristics specific for RA were identified, but were infrequently present. Caution is needed not to overestimate the value of MRI-erosions in the diagnostic process.

\section{Keywords}

Magnetic resonance imaging; erosions; rheumatoid arthritis; age

\section{Introduction}

Rheumatoid arthritis (RA) is characterized by joint inflammation that may lead to bone erosions. Traditionally erosions are evaluated using conventional radiographs. Recently it has been recommended by the EULAR imaging taskforce that magnetic resonance imaging (MRI) is valuable to detect erosions early.[1] Indeed MRI has shown to be more sensitive for structural damage in early RA than conventional radiographs.[2-9]

Radiographic erosions specific for RA are defined in the 2010 ACR/EULAR-criteria[10] as erosions seen in at least three separate joints at the proximal interphalangeal, the metacarpophalangeal(MCP)-, the wrist- and metatarsophalangeal(MTP)-joints (specificity $>80 \%$, sensitivity 15-29\%).[11] However, for MRI-detected erosions a definition specific for RA has not yet been derived. Because MRI is more sensitive in detecting erosions than radiographic imaging, RA-specific MRI-detected erosions need to be characterized.

Previously it was shown that MRI-detected erosions are also observed in other rheumatic diseases and in healthy controls, especially at older age.[12-17] Thus, in order to prevent false-positive MRI-results, it is important to distinguish RA-specific erosions from other erosions.

This cross-sectional study compared erosions in MCP- and MTP-joints as detected on MRI (evaluated using the rheumatoid arthritis MRI scoring system (RAMRIS)) between early RA-patients at the time of diagnosis and symptom-free controls for different characteristics: besides the total erosion score, also the number, severity and location of erosions and the simultaneous presence of MRI-detected inflammation (synovitis and/or bone marrow edema (BME)) were compared. Second, RA-patients were also cross-sectionally compared to early arthritis patients that presented with other diagnoses. Within patients that presented with undifferentiated arthritis (UA), erosions were compared between patients that did and did not progress to RA during the first year. All analyses were done with the ultimate aim to identify features of MRI-detected erosions that are specific for RA. 


\section{Methods}

\section{Patients}

598 patients who presented with early arthritis and were included in the Leiden Early Arthritis Clinic (EAC) between 2010 and 2014 were studied. The EAC is an inception cohort including patients with clinically confirmed arthritis and symptom duration $<2$ years. At baseline questionnaires were administered, joint counts and blood samples were collected and MRI was performed.[18] Nine patients were excluded because no contrast agent was administered. Two weeks after inclusion, when results of regular investigations were known (this did not include information on MRI-results), the initial diagnosis of the patients was documented by the rheumatologists. The clinical diagnosis of RA was verified by fulfilling the 1987- or 2010-criteria at baseline.[10,19] Of the 589 patients 238 patients had RA. The diagnoses of the remaining group with other arthritides $(n=351)$ were UA $(n=192)$, reactive arthritis ( $n=22)$, (pseudo)gout $(n=15)$, psoriatic arthritis $(n=34)$, inflammatory osteoarthritis (OA) $(n=35)$, Lyme arthritis $(n=3)$, paramalignant arthritis $(n=1)$, SLE $(n=4)$, other systemic disorder ( $n=7)$, MCTD, vasculitis $(n=2)$, sarcoidosis $(n=3)$, spondylarthropathy with peripheral arthritis ( $n=5), \operatorname{RS} 3 \mathrm{PE}(\mathrm{n}=10)$, and other diagnosis $(\mathrm{n}=18)$.

In addition, 193 symptom-free controls were recruited by advertisements in local newspapers and websites as previously reported.[20] They had no history of RA or other inflammatory rheumatic diseases, no joint symptoms during the last month, no recent trauma $(<1$ year prior to MRI) and no arthritis at physical examination.

Both studies were approved by the local medical ethics committee. All patients and controls signed informed consent.

\section{MR imaging and scoring}

At baseline MRI of the $2^{\text {nd }}-5^{\text {th }}$ MCP- and $1^{\text {st }}-5^{\text {th }}$ MTP-joints on the most painful side or in case of symmetric symptoms and in healthy controls on the dominant side was performed. MR imaging was performed on a MSK Extreme 1.5T extremity MRI system (General Electric, Wisconsin, USA). The MRIs of all subjects were made on the same scanner. Coronal T1-weighted fast spin-echo (FSE) and contrast-enhanced coronal and axial T1weighted FSE with frequency-selective fat suppression were obtained. Further details on the scan protocol are provided in the Supplementary methods. Erosions, BME and synovitis were scored according to the RAMRIS method, with the exception that BME was assessed on a contrast-enhanced T1-weighted fat-suppressed sequence.[21] According to the RAMRIS method erosions were defined as sharply marginated bone lesions, with correct juxta-articular localization and typical signal characteristics, which are visible in two planes with a cortical break seen in at least one plane. All bones were scored separately for erosions on a scale $0-10$, based on the proportion of eroded bone (0: no erosion, 1: 1-10\% of bone eroded, 2: 11-20\%, etc.). The total erosion score was calculated by summing the erosion score in the MCP- and MTP-joints (range 0-180). Each MRI was scored by two readers, blinded to any clinical data. Intra-reader intraclass correlation coefficients (ICCs) and interreader ICCs were $\searrow 0.86$ (see Supplementary methods). 


\section{Erosion characteristics}

The total erosion score (hence a combination of number of erosions and severity), number, severity and location of erosions were studied on the person level. The presence of concomitant inflammation was studied. This comprised the presence of BME in the same bone or the presence of synovitis around the same bone as where the erosion was located. These analyses were done on person and on bone level. For the total erosion score the mean of two readers was used. When assessing number, severity, location and the combination of erosions with inflammation, MRI-erosions were considered present when the mean of both readers was $\geq 1$ at a specific bone. Grade $\geq 2$ erosions indicate that $>10 \%$ of the bone is eroded.

\section{Statistical analyses}

First, total erosion scores of RA-patients were compared with scores of controls. A linear regression analysis adjusted for age and gender was used with the total erosion score as outcome and group (RA/healthy control) as independent variable. Erosion scores were $\log$ transformed $(\log 10($ score +1$))$ to approximate a normal distribution. The reported effect sizes were back-transformed to the normal score and indicated how many times the erosion scores of RA-patients were higher than that of controls. Thereafter, patients were stratified in three age groups ( $<40,40-59, \succ 60$ years) and frequencies of erosion characteristics were compared between groups. Test characteristics were determined. Similar analyses were performed comparing RA-patients with other arthritides. Finally the diagnostic value of MRI-detected erosions in UA-patients was assessed. SPSS version 23.0 (IBM) was used. Pvalues $<0.05$ were considered significant.

\section{Results}

Patient characteristics

Baseline characteristics of patients and symptom-free controls are presented in Table 1.

\section{At group level RA-patients have slightly higher MRI-detected erosion scores than symptom-free controls but on the individual level there is large overlap}

First the total erosion scores were evaluated. In both the group of RA-patients and that of symptom-free controls the MRI-erosion score was associated with age (Figure 1A, Supplementary table 1). When comparing the erosion scores of RA-patients and controls, RA-patients had 1.20 (95\% CI 1.08-1.33, p<0.001) times higher erosion scores than controls, independent of age and gender. This effect size indicates that RA-patients had in general a $20 \%$ higher total erosion score than controls. Despite the significant difference there was large overlap of MRI-erosion scores between RA-patients and controls, as visually no separate clustering of groups was observed (Figure 1A). Thus total erosion scores could not differentiate RA-patients from controls on the individual level.

\section{Grade $\geq 2$ MRI-erosions are more prevalent in RA-patients than in symptom-free controls}

Then other erosion characteristics were studied to search for RA-specific characteristics. Because of the association with age, all analyses were stratified for age group ( $<40,40-59$ 
and $\geq 60$ years). Since the total erosion score is a combination of the number of erosions and severity, both characteristics were evaluated separately. The median total number of erosions was 1.0 [IQR 0-2.0] for RA-patients and 0 [IQR 0-1.0] for symptom-free controls (MannWhitney U-test: $\mathrm{p}=0.001$ ). Within the different age groups there were no significant differences in the two oldest groups. In the group $<40$ years, RA-patients had more erosions than controls (median 0 [IQR 0-1.0] versus 0 [IQR 0-0], $\mathrm{p}=0.007$ ) though differences were too small to identify a number of MRI-detected erosions that was specific for RA. To determine whether grade $\geq 2$ erosions were RA-specific, the frequency of grade $\geq 2$ erosions was considered per joint location (Supplementary table 2). This revealed that grade $\geq 2$ erosions were almost exclusively present in RA (specificity $98-100 \%$ for different age groups, Table 2). However within all age groups they were only sporadically observed in RA at disease presentation (sensitivity 5-10\%). Evaluation on person level showed that $8 \%$ of the RA-patients had at least one grade $\geq 2$ erosion in an MCP- and/or MTP-joint, while in controls this was only $1 \%$ (Table 3 ). Thus the presence of grade $\geq 2$ erosions was highly specific for RA, but also infrequent in RA at disease presentation.

\section{MTP-5 and MTP-1 are more often affected in RA-patients than in symptom-free controls}

Then the location (the affected MCP- or MTP-joint) was assessed (Table 4). Both in RApatients and controls most erosions were located in the proximal part of the MCP- and MTPjoints: in RA-patients $82-95 \%$ of the erosions was located proximal in the joint and in controls this was $81-100 \%$ for the different age groups. As presented in Table 4, overall the MCP- and MTP-bones that were frequently affected in RA-patients were also frequently affected in healthy controls. For instance MCP2 and MCP3 were predilection sites for MRIdetected erosions in RA, but also in controls. However there were also some differences: erosions in MTP5 were more frequently present in RA-patients than in controls in most age groups (specificity 90-98\% for different age groups, Table 2). In addition, erosions in MTP1 in the age group <40 almost exclusively occurred in RA (specificity $98 \%$ ); the specificity was lower in older age groups (specificity $86 \%$ if aged $40-59$ and $63 \%$ if aged $\geq 60$ ).

Examples of MRI-detected erosions are shown in Figure 2.

\section{Erosions with the simultaneous presence of BME and/or synovitis are more frequent in RA-patients than in symptom-free controls}

Then we questioned whether the combined presence of erosions with surrounding inflammation was specific for RA. At bone level, in RA-patients 33\% (95/285) of the total number of MCP- and MTP-bones with erosions only had erosions without synovitis and/or BME while in controls this was 77\% (105/136, Table 5). Similarly, when analysed on person level, $16 \%$ of the RA-patients only had erosions without inflammation and $40 \%$ had at least one erosion with inflammation in that same joint while in controls this was 30\% and 12\%, respectively (Table 3 ). When analysing the different age groups it appeared that within the age group $<40$ years, the simultaneous presence of erosions with inflammation was exclusively observed in RA-patients (specificity 100\%). In the age group 40-59 years the specificity was $91 \%$ and it was lower in persons aged $\succeq 60$ (specificity $71 \%$ ) since in this age group erosions with inflammation were also observed in healthy controls (Table 2). Thus the presence of erosions with inflammation was specific for RA, but only if aged $<60$. 
Altogether, the presence of grade $\geq 2$ erosions and MTP5-erosions were specific for RA in all age groups, erosions with inflammation were specific for RA if aged $<60$ and MTP1erosions if aged $<40$. Although these erosion characteristics were highly specific for RA only $29 \%$ of all RA-patients had $\geq 1$ erosion(s) with $\geq 1$ of these characteristics.

\section{Erosions in MTP5 and grade $\geq 2$ erosions in all age groups and erosions in MTP1 if aged $<40$ remain specific for $\mathrm{RA}$ when compared to patients with other arthritides}

Thus far, different erosion characteristics were compared between RA-patients and controls revealing some RA-specific characteristics. The next question is whether these characteristics are truly RA-specific or are also present in other arthritides. Therefore, all analyses were repeated with patients with other arthritides as reference group. The total erosion scores of both patient groups were not significantly different (beta 0.92 95\%CI 0.84-1.01, Figure 1B). Comparison of the different erosion characteristics showed that the presence of grade $\geq 2$ erosions was RA-specific in all age groups (specificity $100 \%$ if aged $<40$ and $96 \%$ if aged $40-59$ and $\succ 60$, Table 2, Supplementary table 3). Also MTP5-erosions were highly specific for RA in all age groups (specificity $100 \%$ if aged $<40,89 \%$ if aged 40-59 and $90 \%$ if aged $\ 60$, Table 2 , Supplementary table 4). The specificity of MTP1erosions was $93 \%$ in patients aged $<40$ but at higher age specificity decreased to $66 \%$. Erosions with inflammation were less specific for RA (specificity 49-85\% within different age groups) as these were also present in other arthritides. Thus, erosions with inflammation were not RA-specific, but MTP5-erosions and grade $\geq 2$ erosions were specific in all age groups and MTP1-erosions in patients aged $<40.21 \%$ of RA-patients had $\geq 1$ erosion(s) with these characteristics (sensitivity $21 \%$ ). Additionally, of all patients with erosions with one of these three finally identified features, 53\% fulfilled criteria for RA (PPV 53\%) and of all patients without such erosions, criteria were not fulfilled in 62\% (NPV 62\%).

\section{MRI-detected erosions do not contribute to the identification of UA-patients that will progress to RA}

Finally, the value of MRI-detected erosions was evaluated within UA-patients. Of the UApatients, $15 \%$ (28/192) fulfilled criteria for RA after one year. Of these patients $11 \%$ had an RA-specific erosion at baseline, whereas $9 \%$ of the non-convertors had an RA-specific erosion (OR 1.3 95\%CI 0.3-4.8).

\section{Discussion}

Radiographic erosions specific for RA have been defined as the presence of $\geq 3$ radiographic erosions on MCP-, PIP-, wrist- or MTP-joints and their presence is considered sufficiently specific to classify RA.[11] MRI is a sensitive imaging modality that depicts cortical defects and therefore is suitable to detect erosive damage. Thus far it was unknown which MRIdetected erosions on hand and foot joints are specific for RA. This large cross-sectional MRI-study showed that on the group level, RA-patients had higher MRI-detected erosion scores in MCP- and MTP-joints than controls, but also that there was large overlap on the individual level. Several erosion characteristics were studied in detail; this was done within three age strata as the total MRI-erosion score was associated with age. Compared to controls from the general population, four characteristics were identified as RA-specific: 
grade $\geq 2$ erosions, MTP5-erosions, MTP1-erosions if aged $<40$ and erosions with local inflammation if aged $<60$. At least one of these characteristics is present in $29 \%$ of RApatients.

Subsequently RA-patients were compared to early arthritis patients with other diagnoses, because studies comparing established cases and healthy controls will reveal the maximal contrast. Differences are often smaller when more clinically relevant patient groups are studied.[22,23] Indeed we observed that some erosion characteristics that were specific for RA when compared to controls were not specific when RA was compared with other arthritides. This was most prominent for the combined presence of erosions with inflammation. Nonetheless, some characteristics (grade $\geq 2$ erosions, MTP5-erosions, MTP1erosions in persons aged $<40$ ) were RA-specific in both settings. $21 \%$ of RA-patients had $\geq 1$ erosion(s) with $\geq 1$ of these characteristics.

Although some erosion characteristics were identified as RA-specific, an important overlap between early RA-patients and controls was observed. It has been recommended that novel imaging modalities, such as MRI, can be used to detect erosions early.[1] The present data show that if all MRI-detected erosions (according to RAMRIS) would be considered as characteristic for RA or disease, this would yield many false-positive results.

We used the RAMRIS-definition of erosions that basically evaluated the volume of the erosion in relation to the assessed bone. Others showed that small lesions on high-resolution peripheral quantitative computed tomography were not entirely specific for RA and suggested that lesions $>1.9 \mathrm{~mm}$ in diameter were highly specific.[24,25] It remains to be determined if a phenotypic definition of MRI-erosions, for instance one that includes a description of the size of the cortical break, will be more discriminative; this is subject of further studies and is also considered within an ongoing EULAR taskforce.

Some of the findings on MRI-detected erosions are in line with previous findings on radiographic erosions. Radiographic erosions have been shown to occur more frequently at disease onset with higher age.[26-32] MTP5 has been shown as a predilection site for RArelated erosions as well.[33-35]. We observed that the large majority of erosions (both in RA and in the other groups studied) were located in the proximal bone of the joint which is completely in line with previous findings.[24,34,36]

Erosive lesions in symptom-free controls have also been reported in other studies.[12] The nature of these lesions is unclear. Because of the association with age, degenerative subchondral bone cysts may be one of the explanations. In addition a very recent study, evaluating bone microstructure of MCP-joints using high-resolution tomography and micro$\mathrm{CT}$, demonstrated that the number of so called cortical microchannels (linking the synovial and bone marrow compartments) was higher in RA-patients than in healthy controls and was associated with erosions and with age.[37] It is intriguing to speculate that these channels have a causal role in erosion development, both in RA and controls. Another possibility is that mechanical strains are involved in erosion development, since erosions were frequently located in the foot (49\% of the erosions in RA-patients and 38\% in symptom-free controls). 
However, a pathophysiological explanation for the findings done in symptom-free persons is beyond the scope of this study.

The location of erosions within the bone was not studied here. This information could not be discerned using RAMRIS as this method evaluates the volume of the erosive lesion per bone. However, previous studies have shown that the majority of erosive MRI-lesions in MCP-joints occurred adjacent to the radial collateral ligaments, both in RA-patients and in healthy controls.[38,39] Similar observations were done in a study in RA-patients and healthy controls on the location of erosions as detected on CT.[24] The location of erosions in the symptom-free controls that were studied here has been reported previously[13], and showed that also in these persons erosions were present adjacent to the collateral ligaments and were not situated centrally in the bone. Because of these previous reports, showing no difference in location of erosions within the bone between RA-patients and controls, we anticipated that this characteristic will not result in further discrimination of RA-specific erosions from other erosions.

Cross-sectional analyses revealed that of all patients with an erosion that was identified as characteristic for RA 53\% actually had RA (PPV). Likewise, $62 \%$ of all patients without such erosions did not have RA (NPV), whereas $38 \%$ did fulfil criteria for RA. These data illustrate that the absence or presence of RA-specific erosions at disease presentation are of moderate value to identify patients that fulfil criteria for RA at the same point in time.

Longitudinal analysis within UA-patients suggested that the presence of RA-specific erosions was also not predictive for the development of RA. However, this analysis was of limited power. Additionally, other outcomes, such as the start of DMARDs, should be studied, since DMARD-treatment might hamper progression to RA. Finally, it was not possible to study the different RA-specific erosion features separately due to the limited number of patients. Further studies are warranted.

We studied an early RA-population. $36 \%$ of the patients was RF negative and $48 \%$ were ACPA negative which is comparable to other early RA cohorts.[40,41] Our population is somewhat different from RA-patients included in clinical trials where generally a selection of RA-patients is included.

A limitation of this study is that it was cross-sectional in nature and that imaging follow-up was not studied. Sensitivity of readers could have been a problem and could be equally present in the three groups. The presence of serial MRI-data facilitates the differentiation of erosions from vascular channels and entheseal attachments, as these should not change during follow-up. Erosions in contrast could progress over time, although this progression may also have been hampered by up-to-date treatment strategies. Serial MRIs were not made but would have been beneficial to evaluate if some erosions were falsely identified as such. However if MRI will be used for early identification of patients in clinical practice, single MRI-measurements will be made.

In conclusion, MRI-detected erosions (according to the RAMRIS definition) in MCP- and MTP-joints are not confined to RA, but also present in other arthritides and in symptom-free persons from the general population. On the individual level there was a large overlap. Some 
erosion characteristics were identified as specific for RA (grade $\geq 2$ erosions, MTP5erosions, and MTP1-erosions if aged $<40)$, though these occurred in a minority $(21 \%)$ of the patients. Longitudinal MR-imaging may improve specificity; however this was not tested in this study. The present data imply that if single measurements with novel imaging modalities such as MRI are used for the early detection of structural damage in clinical practice, the risk of false-positive findings should be considered.

\section{Supplementary Material}

Refer to Web version on PubMed Central for supplementary material.

\section{Acknowledgements}

The authors would like to thank G. Kracht from the Department of Radiology of the Leiden University Medical Center for processing the MRIs. This paper is based on work that was previously presented at the 2017 ACR/ARHP Annual Meeting; November 7, 2017; San Diego, California; and was published as a conference abstract: Boeters DM et al, Arthritis Rheumatol. 2017; 69 (suppl 10).

\section{Funding}

This project has received funding from the European Research Council (ERC) under the European Union's Horizon 2020 research and innovation programme (Starting grant, agreement No 714312) and from the Netherlands Organization for Health Research and Development (Vidi grant).

\section{References}

1. Colebatch AN, Edwards CJ, Østergaard M, et al. EULAR recommendations for the use of imaging of the joints in the clinical management of rheumatoid arthritis. Ann Rheum Dis. 2013; 72:804-14. DOI: 10.1136/annrheumdis-2012-203158 [PubMed: 23520036]

2. Backhaus M, Kamradt T, Sandrock D, et al. Arthritis of the finger joints: A comprehensive approach comparing conventional radiography, scintigraphy, ultrasound, and contrast-enhanced magnetic resonance imaging. Arthritis Rheum. 1999; 42:1232-45. DOI: 10.1002/1529-0131(199906)42:6<1232::AID-ANR21>3.0.CO;2-3 [PubMed: 10366117]

3. McQueen FM, Stewart N, Crabbe J, et al. Magnetic resonance imaging of the wrist in early rheumatoid arthritis reveals a high prevalence of erosions at four months after symptom onset. Ann Rheum Dis. 1998; 57:350-6. [PubMed: 9771209]

4. Gilkeson G, Polisson R, Sinclair H, et al. Early detection of carpal erosions in patients with rheumatoid arthritis: a pilot study of magnetic resonance imaging. J Rheumatol. 1988; 15:1361-6. [PubMed: 3199397]

5. Backhaus M, Burmester GR, Sandrock D, et al. Prospective two year follow up study comparing novel and conventional imaging procedures in patients with arthritic finger joints. Ann Rheum Dis. 2002; 61:895-904. [PubMed: 12228160]

6. Klarlund M, Ostergaard M, Jensen KE, et al. Magnetic resonance imaging, radiography, and scintigraphy of the finger joints: one year follow up of patients with early arthritis. The TIRA Group. Ann Rheum Dis. 2000; 59:521-8. [PubMed: 10873961]

7. Foley-Nolan D, Stack JP, Ryan M, et al. Magnetic resonance imaging in the assessment of rheumatoid arthritis--a comparison with plain film radiographs. Br J Rheumatol. 1991; 30:101-6. [PubMed: 2012935]

8. Lindegaard H, Vallø J, Hørslev-Petersen K, et al. Low field dedicated magnetic resonance imaging in untreated rheumatoid arthritis of recent onset. Ann Rheum Dis. 2001; 60:770-6. DOI: 10.1136/ ard.60.8.770 [PubMed: 11454641]

9. Ejbjerg BJ, Vestergaard A, Jacobsen S, et al. Conventional radiography requires a MRI-estimated bone volume loss of $20 \%$ to $30 \%$ to allow certain detection of bone erosions in rheumatoid arthritis 
metacarpophalangeal joints. Arthritis Res Ther. 2006; 8:R59.doi: 10.1186/ar1919 [PubMed: 16542505]

10. Aletaha D, Neogi T, Silman AJ, et al. 2010 rheumatoid arthritis classification criteria: an American College of Rheumatology/European League Against Rheumatism collaborative initiative. Ann Rheum Dis. 2010; 69:1580-8. DOI: 10.1136/ard.2010.138461 [PubMed: 20699241]

11. van der Heijde D, van der Helm-van Mil AHM, Aletaha D, et al. EULAR definition of erosive disease in light of the 2010 ACR/EULAR rheumatoid arthritis classification criteria. Ann Rheum Dis. 2013; 72:479-81. DOI: 10.1136/annrheumdis-2012-202779 [PubMed: 23378540]

12. Mangnus L, Schoones JW, van der Helm-van Mil AHM. What is the prevalence of MRI-detected inflammation and erosions in small joints in the general population? A collation and analysis of published data. RMD Open. 2015; 1:e000005.doi: 10.1136/rmdopen-2014-000005 [PubMed: 26509042]

13. Mangnus L, van Steenbergen HW, Reijnierse M, et al. Magnetic Resonance Imaging-Detected Features of Inflammation and Erosions in Symptom-Free Persons From the General Population. Arthritis Rheumatol. 2016; 68:2593-602. DOI: 10.1002/art.39749 [PubMed: 27213695]

14. Boutry N, Hachulla E, Flipo R-M, et al. MR imaging findings in hands in early rheumatoid arthritis: comparison with those in systemic lupus erythematosus and primary Sjögren syndrome. Radiology. 2005; 236:593-600. DOI: 10.1148/radiol.2361040844 [PubMed: 15972342]

15. Olech E, Crues JV, Yocum DE, et al. Bone Marrow Edema Is the Most Specific Finding for Rheumatoid Arthritis (RA) on Noncontrast Magnetic Resonance Imaging of the Hands and Wrists: A Comparison of Patients with RA and Healthy Controls. J Rheumatol. 2010; 37:265-74. DOI: 10.3899/jrheum.090062 [PubMed: 19955056]

16. Tani C, Chiara T, D'Aniello D, et al. MRI pattern of arthritis in systemic lupus erythematosus: a comparative study with rheumatoid arthritis and healthy subjects. Skeletal Radiol. 2015; 44:261-6. DOI: 10.1007/s00256-014-2033-0 [PubMed: 25341505]

17. Ejbjerg B, Narvestad E, Rostrup E, et al. Magnetic resonance imaging of wrist and finger joints in healthy subjects occasionally shows changes resembling erosions and synovitis as seen in rheumatoid arthritis. Arthritis Rheum. 2004; 50:1097-106. DOI: 10.1002/art.20135 [PubMed: 15077291]

18. de Rooy DPC, van der Linden MPM, Knevel R, et al. Predicting arthritis outcomes-what can be learned from the Leiden Early Arthritis Clinic? Rheumatology. 2011; 50:93-100. DOI: 10.1093/ rheumatology/keq230 [PubMed: 20639266]

19. Arnett FC, Edworthy SM, Bloch DA, et al. The american rheumatism association 1987 revised criteria for the classification of rheumatoid arthritis. Arthritis Rheum. 1988; 31:315-24. DOI: 10.1002/art.1780310302 [PubMed: 3358796]

20. Mangnus L, van Steenbergen Hw, Reijnierse M, et al. MR-detected features of inflammation and erosions occur in symptom-free persons from the general population. Arthritis Rheumatol. 2016; doi: 10.1002/art.39749

21. Østergaard M, Peterfy C, Conaghan P, et al. OMERACT Rheumatoid Arthritis Magnetic Resonance Imaging Studies. Core set of MRI acquisitions, joint pathology definitions, and the OMERACT RA-MRI scoring system. J Rheumatol. 2003; 30:1385-6. [PubMed: 12784422]

22. Schünemann HJ, Oxman AD, Brozek J, et al. GRADE: assessing the quality of evidence for diagnostic recommendations. Evid Based Med. 2008; 13:162-3. DOI: 10.1136/ebm.13.6.162-a [PubMed: 19043023]

23. Rutjes AWS, Reitsma JB, Di Nisio M, et al. Evidence of bias and variation in diagnostic accuracy studies. CMAJ Can Med Assoc J J Assoc Medicale Can. 2006; 174:469-76. DOI: 10.1503/cmaj. 050090

24. Stach CM, Bäuerle M, Englbrecht M, et al. Periarticular bone structure in rheumatoid arthritis patients and healthy individuals assessed by high-resolution computed tomography. Arthritis Rheum. 2010; 62:330-9. DOI: 10.1002/art.27252 [PubMed: 20112404]

25. Barnabe C, Toepfer D, Marotte H, et al. Definition for Rheumatoid Arthritis Erosions Imaged with High Resolution Peripheral Quantitative Computed Tomography and 28 Interreader Reliability for Detection and Measurement. J Rheumatol. 2016; 43:1935-40. DOI: 10.3899/jrheum.160648 [PubMed: 27698108] 
26. Mangnus L, van Steenbergen HW, Lindqvist E, et al. Studies on ageing and the severity of radiographic joint damage in rheumatoid arthritis. Arthritis Res Ther. 2015; 17doi: 10.1186/ s13075-015-0740-0

27. Innala L, Berglin E, Möller B, et al. Age at onset determines severity and choice of treatment in early rheumatoid arthritis: a prospective study. Arthritis Res Ther. 2014; 16:R94.doi: 10.1186/ ar4540 [PubMed: 24731866]

28. Bukhari M, Lunt M, Barton A, et al. Increasing age at symptom onset is associated with worse radiological damage at presentation in patients with early inflammatory polyarthritis. Ann Rheum Dis. 2007; 66:389-93. DOI: 10.1136/ard.2006.055657 [PubMed: 16950810]

29. Calvo-Alén J, Corrales A, Sánchez-Andrada S, et al. Outcome of late-onset rheumatoid arthritis. Clin Rheumatol. 2005; 24:485-9. DOI: 10.1007/s10067-004-1067-4 [PubMed: 15750680]

30. Khanna D, Ranganath VK, Fitzgerald J, et al. Increased radiographic damage scores at the onset of seropositive rheumatoid arthritis in older patients are associated with osteoarthritis of the hands, but not with more rapid progression of damage. Arthritis Rheum. 2005; 52:2284-92. DOI: 10.1002/art.21221 [PubMed: 16052588]

31. Kuiper S, van Gestel AM, Swinkels HL, et al. Influence of sex, age, and menopausal state on the course of early rheumatoid arthritis. J Rheumatol. 2001; 28:1809-16. [PubMed: 11508583]

32. van der Heijde DM, van Riel PL, van Leeuwen MA, et al. Older versus younger onset rheumatoid arthritis: results at onset and after 2 years of a prospective followup study of early rheumatoid arthritis. J Rheumatol. 1991; 18:1285-9. [PubMed: 1757926]

33. Hulsmans HM, Jacobs JW, van der Heijde DM, et al. The course of radiologic damage during the first six years of rheumatoid arthritis. Arthritis Rheum. 2000; 43:1927-40. DOI: 10.1002/1529-0131(200009)43:9<1927::AID-ANR3>3.0.CO;2-B [PubMed: 11014342]

34. Siddle HJ, Hensor EMA, Hodgson RJ, et al. Anatomical location of erosions at the metatarsophalangeal joints in patients with rheumatoid arthritis. Rheumatol Oxf Engl. 2014; 53:932-6. DOI: 10.1093/rheumatology/ket478

35. Tuna H, Birtane M, Taştekin N, et al. Pedobarography and its relation to radiologic erosion scores in rheumatoid arthritis. Rheumatol Int. 2005; 26:42-7. DOI: 10.1007/s00296-004-0504-7 [PubMed: 15449023]

36. Peterfy CG, Countryman P, Gabriele A, et al. Magnetic Resonance Imaging in Rheumatoid Arthritis Clinical Trials: Emerging Patterns Based on Recent Experience. J Rheumatol. 2011; 38:2023-30. DOI: 10.3899/jrheum.110418 [PubMed: 21885511]

37. Werner D, Simon D, Englbrecht M, et al. Early Changes of the Cortical Micro-Channel System in the Bare Area of the Joints of Patients With Rheumatoid Arthritis. Arthritis Rheumatol Hoboken NJ. 2017; 69:1580-7. DOI: 10.1002/art.4014829

38. Tan AL, Tanner SF, Conaghan PG, et al. Role of metacarpophalangeal joint anatomic factors in the distribution of synovitis and bone erosion in early rheumatoid arthritis. Arthritis Rheum. 2003; 48:1214-22. DOI: 10.1002/art.10963 [PubMed: 12746894]

39. Palosaari K, Vuotila J, Soini I, et al. Small bone lesions resembling erosions can frequently be found in bilateral wrist MRI of healthy individuals. Scand J Rheumatol. 2009; 38:450-4. DOI: 10.3109/03009740903002257 [PubMed: 19922020]

40. Ajeganova S, Humphreys JH, Verheul MK, et al. Anticitrullinated protein antibodies and rheumatoid factor are associated with increased mortality but with different causes of death in patients with rheumatoid arthritis: a longitudinal study in three European cohorts. Ann Rheum Dis. 2016; 75:1924-32. DOI: 10.1136/annrheumdis-2015-208579 [PubMed: 26757747]

41. Roubille C, Rincheval N, Dougados M, et al. Seven-year tolerability profile of glucocorticoids use in early rheumatoid arthritis: data from the ESPOIR cohort. Ann Rheum Dis. 2017; 76:1797-802. DOI: 10.1136/annrheumdis-2016-210135 [PubMed: 28213564] 


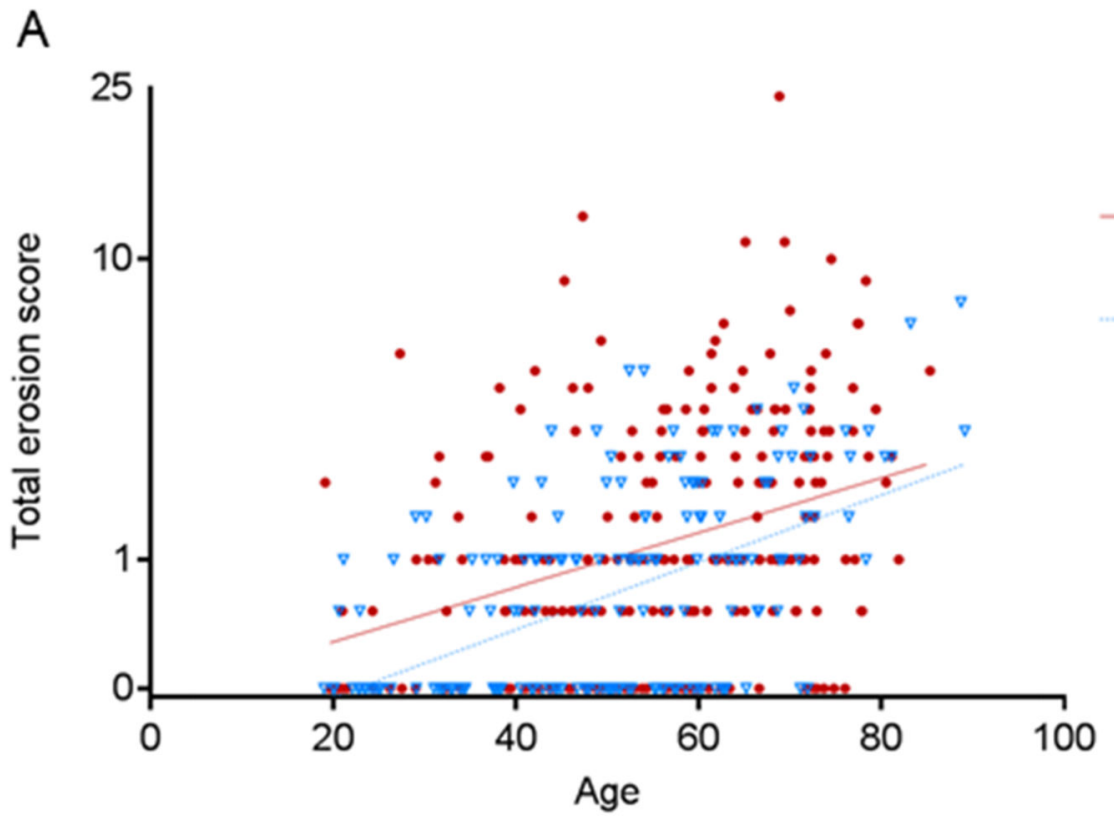

- Rheumatoid arthritis patients

$\checkmark$ Symptom-free controls

Regression line rheumatoid arthritis patients Regression line symptom-free controls

B

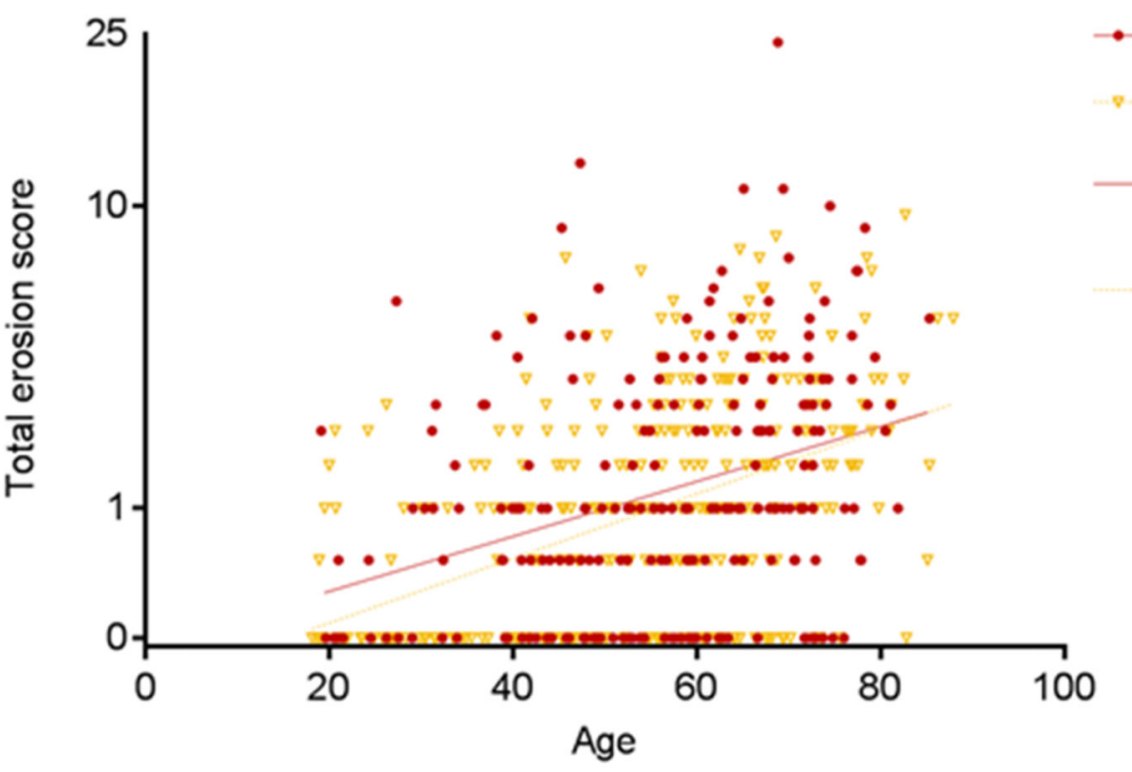

- Rheumatoid arthritis patients Other arthritis patients Regression line rheumatoid arthritis patients Regression line other arthritis patients

Figure 1. MRI-detected erosions in MCP- and MTP-joints in relation to age in RA-patients and in controls $(A)$ and in patients with other arthritides (B); both figures show overlap at the individual level

Linear regression analyses were performed with the mean total erosion score as detected by magnetic resonance imaging as outcome and group (RA-patients and healthy controls (A) or other arthritides (B)), age and gender as independent variables. Y-axis is log-transformed. 

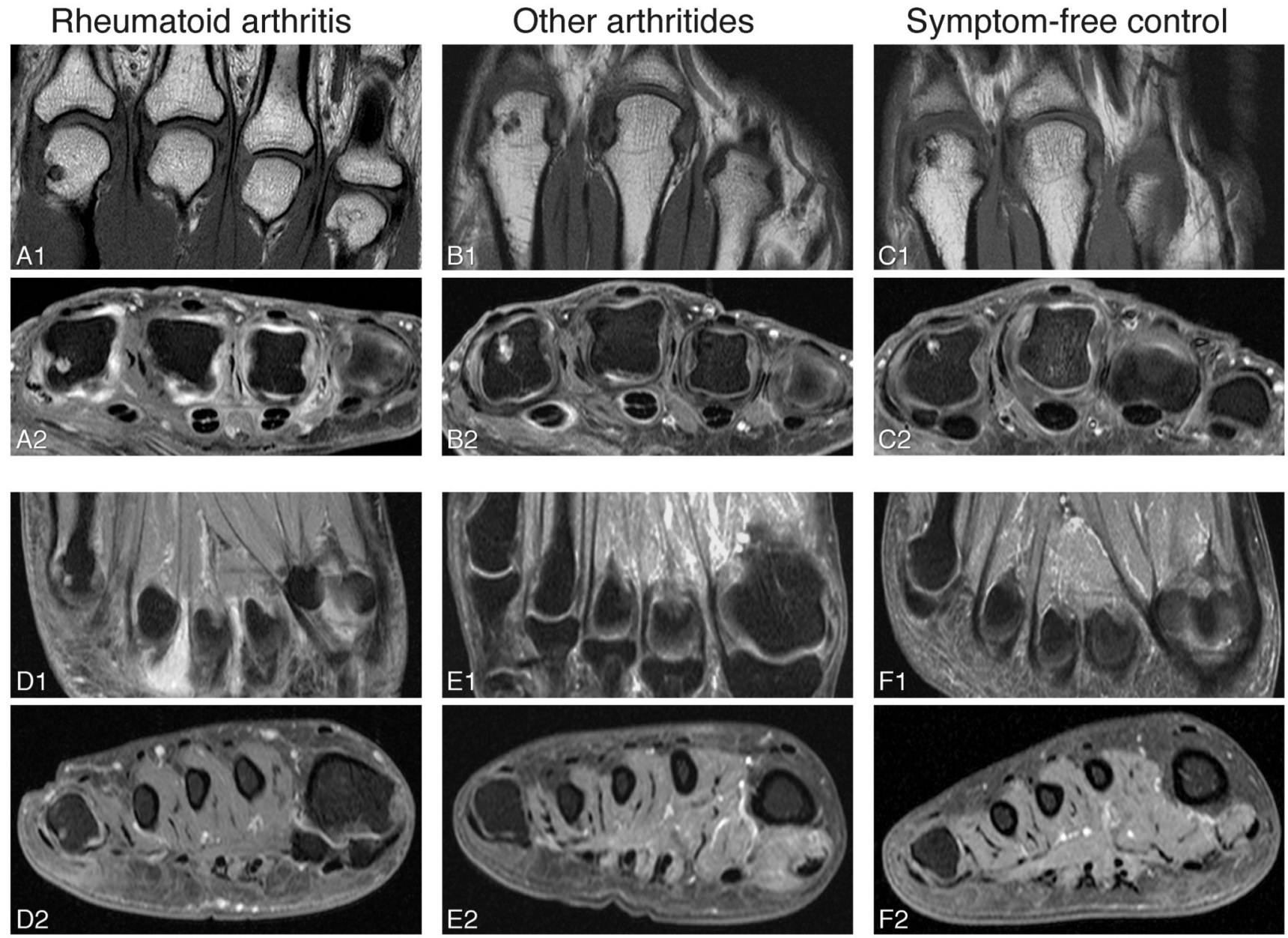

Figure 2. Examples of erosions in RA-patients, patients with other arthritides and symptom-free controls

MR images of RA-patients (A, D), patients with other arthritides (B, E) and symptom-free controls (C, F). Examples of erosion in MCP2 (A-C) and MTP5 are shown (D). Erosions in MCP2 were observed in all different groups (A-C), while erosions in MTP5 were mainly observed in RA-patients (D). The erosion shown in MTP5 (D) is accompanied by the presence of bone marrow edema. Patient $\mathrm{B}$ was diagnosed with gout. Person $\mathrm{C}$ was aged 48 years. Coronal (A1, B1, C1, D1, E1, F1) and axial (A2, B2, C2, D2, E2, F2) images are shown. MRI sequences included coronal T1-weighted fast spin-echo (FSE) sequences and axial T1-weighted FSE sequences with fat suppression after contrast enhancement. RA, rheumatoid arthritis; MCP, metacarpophalangeal joint; MTP, metatarsophalangeal joint; MRI, magnetic resonance imaging. 


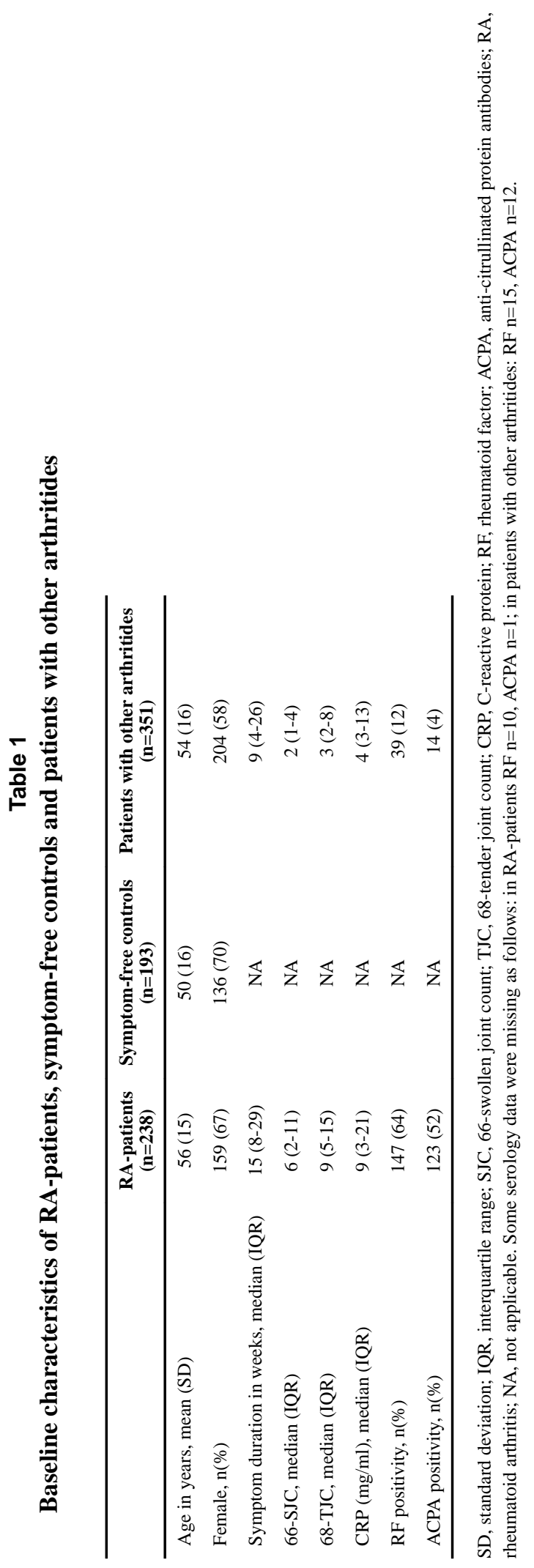




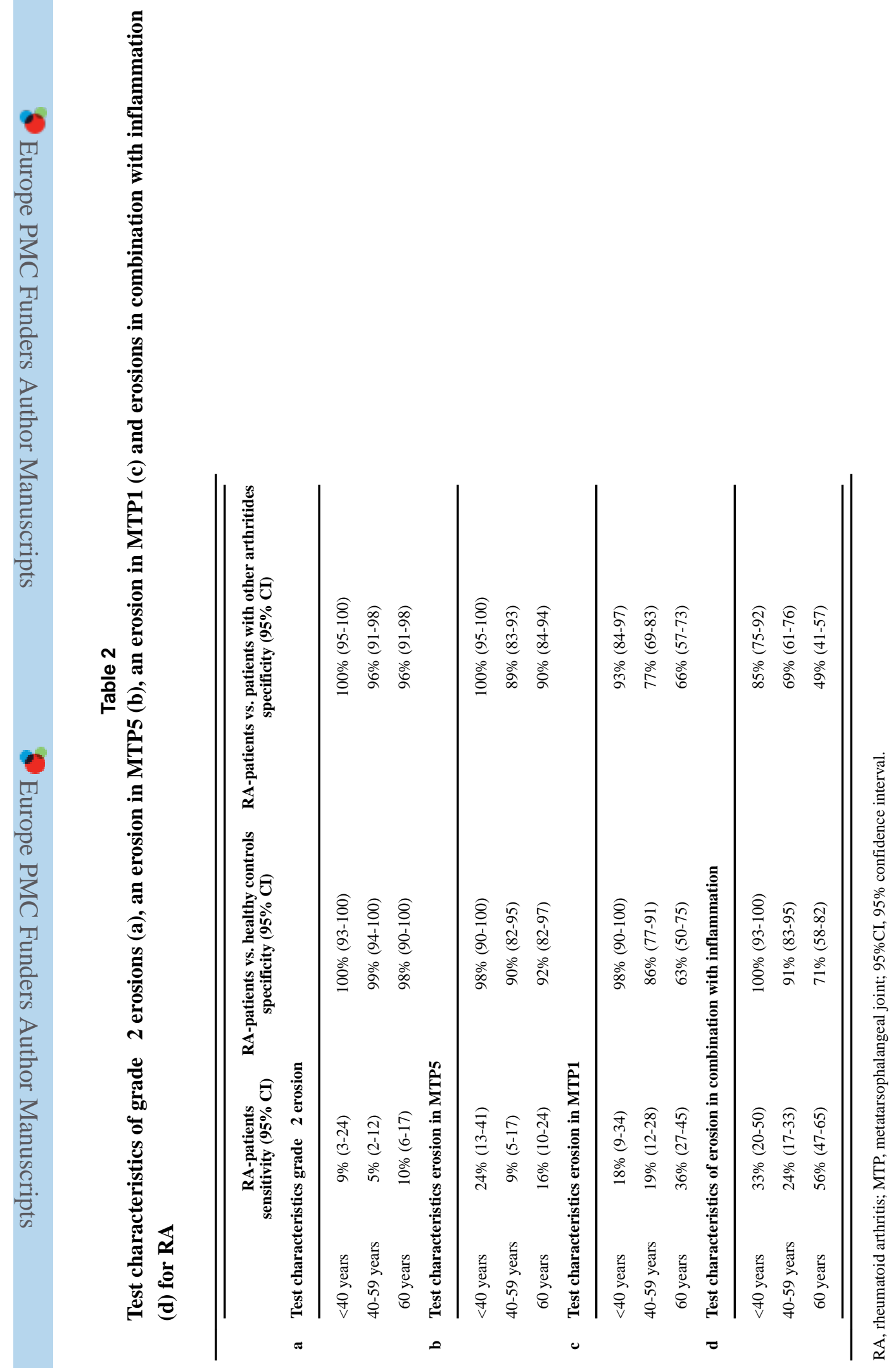




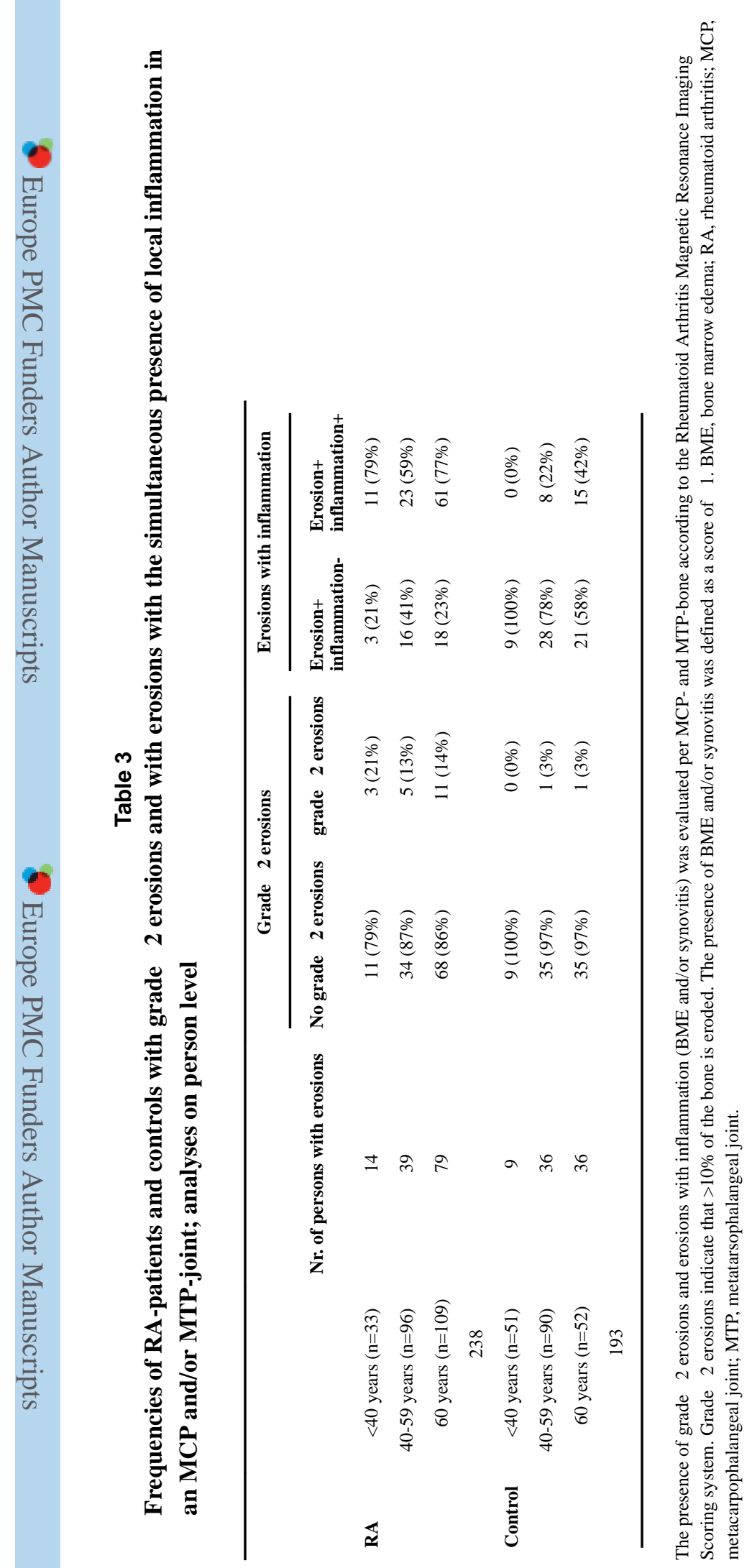

Ann Rheum Dis. Author manuscript; available in PMC 2019 March 05. 
Table 4

Location of erosions in bones of the MCP- and MTP-joints of RA-patients and symptom free controls, depicted per age category $(18-39,40-59, \geq 60$ years)

\begin{tabular}{|c|c|c|c|c|c|c|c|c|}
\hline \multirow[b]{2}{*}{ Erosions } & & \multicolumn{2}{|c|}{$<40$ years } & \multicolumn{2}{|c|}{ 40-59 years } & \multicolumn{2}{|c|}{$\mathbf{7 0} 0$ years } & \\
\hline & & $\underset{\mathbf{n}=\mathbf{3 3}}{\mathbf{R A}}$ & $\begin{array}{c}\text { Control } \\
\mathbf{n}=51\end{array}$ & $\underset{\mathrm{n}=96}{\mathrm{RA}}$ & $\begin{array}{c}\text { Control } \\
\mathbf{n}=90\end{array}$ & $\underset{n=109}{R A}$ & $\begin{array}{c}\text { Control } \\
\mathbf{n}=52\end{array}$ & \\
\hline \multirow[t]{2}{*}{ MCP 2} & proximal & 6 & 6 & 7 & 11 & 23 & 23 & \\
\hline & distal & 0 & 0 & 4 & 2 & 9 & 10 & \\
\hline \multirow[t]{2}{*}{ MCP 3} & proximal & 9 & 8 & 15 & 12 & 30 & 23 & \\
\hline & distal & 0 & 0 & 0 & 1 & 5 & 4 & Percentage \\
\hline \multirow[t]{2}{*}{$\mathrm{MCP} 4$} & proximal & 3 & 0 & 3 & 2 & 9 & 8 & 40 \\
\hline & distal & 0 & 0 & 0 & 0 & 0 & 0 & \\
\hline \multirow[t]{2}{*}{ MCP 5} & proximal & 0 & 2 & 5 & 6 & 20 & 13 & 30 \\
\hline & distal & 0 & 0 & 0 & 0 & 1 & 6 & \\
\hline \multirow[t]{2}{*}{ MTP 1} & proximal & 18 & 2 & 19 & 14 & 36 & 37 & 20 \\
\hline & distal & 0 & 0 & 2 & 0 & 11 & 6 & 10 \\
\hline \multirow[t]{2}{*}{ MTP 2} & proximal & 0 & 0 & 2 & 0 & 4 & 0 & \\
\hline & distal & 3 & 0 & 0 & 1 & 4 & 0 & \\
\hline \multirow[t]{2}{*}{ MTP 3} & proximal & 0 & 0 & 2 & 0 & 6 & 0 & \\
\hline & distal & 0 & 0 & 0 & 0 & 2 & 0 & \\
\hline \multirow[t]{2}{*}{ MTP 4} & proximal & 3 & 0 & 3 & 0 & 1 & 0 & \\
\hline & distal & 0 & 0 & 0 & 0 & 0 & 0 & \\
\hline \multirow[t]{2}{*}{ MTP 5} & proximal & 24 & 2 & 9 & 10 & 16 & 8 & \\
\hline & distal & 0 & 0 & 2 & 0 & 1 & 2 & \\
\hline
\end{tabular}

Values are the percentages of persons with an erosion of all persons in that age category. The presence of an erosion is defined as an erosion score of at least 1 in that bone. RA, rheumatoid arthritis; MCP, metacarpophalangeal joint; MTP, metatarsophalangeal joint. 


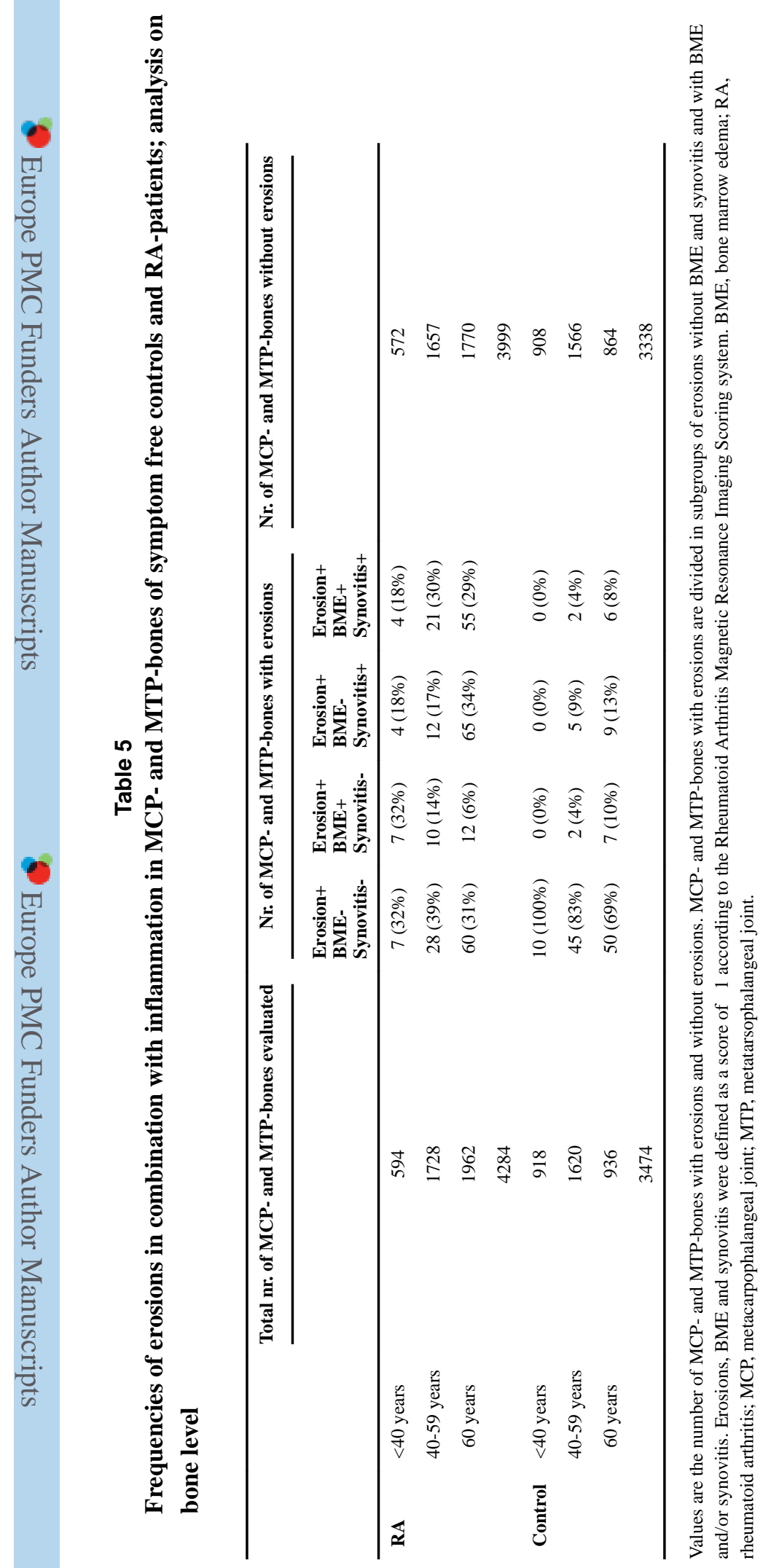

Ann Rheum Dis. Author manuscript; available in PMC 2019 March 05. 